\title{
Dedicato ad Amedeo De Vecchi
}

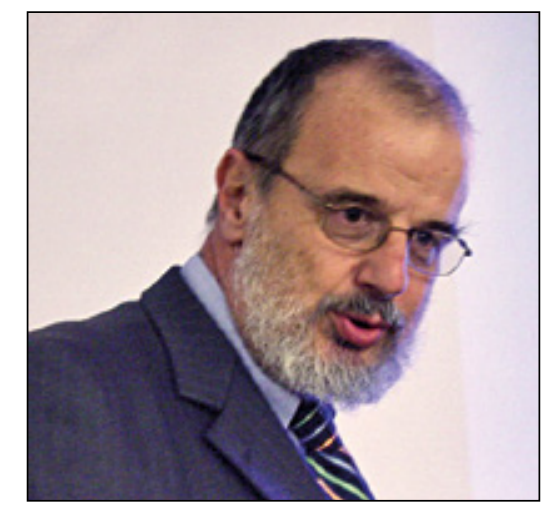

Ogni volta che lo incontravo ero contenta: sentivo per Lui affetto e gratitudine.

Amedeo è stato importante per me: è stato Lui, infatti, a offrirmi l'opportunità di coordinare il GdSDP, un'esperienza che ha segnato in positivo la mia vita lavorativa e che si è rivelata cruciale per il mio cammino successivo.

Amedeo trasmetteva sicurezza e solidità e, nello stesso tempo, disponibilità e condivisione.

Insieme a pochi altri è stato un punto di riferimento nel mio percorso di formazione in dialisi peritoneale e, quando lo consultavo, la risposta arrivava pronta e sembrava tanto semplice quanto giusta.

Il fatto che non ci sia più e che non lo potrò più rivedere mi dà tanta, tanta tristezza.

Grazie, Amedeo!

Flavia Caputo

La mia amicizia con Amedeo De Vecchi risale alla fine degli anni '70 ed è stata solida, come quelle che nascono tra vecchi commilitoni. In quegli anni, ambedue lavoravamo in ambienti che condividevano in qualche misura la provocazione di Shaldon: "La DP è terapia di serie B per medici di serie B". L'amicizia si è, poi, rinforzata per la comune "militanza" nel Gruppo Cooperativo e nel Gruppo di Studio della DP.

Oltre che di lavoro, si parlava spesso anche delle nostre famiglie e il comune sentire su questo tema è servito a consolidare il nostro legame.

Amedeo era tra i pochissimi in grado di concepire autonomamente ricerche originali e di avere contatti personali con Colleghi stranieri di primo piano.

Quando decise di lasciare l'Ospedale, era molto amareggiato e, in quel periodo, per un po' ci siamo sentiti con maggiore frequenza. Seppe presto reagire e prevalse la sua passione per il lavoro e, in particolare, per la dialisi peritoneale.

Per una serie di circostanze, ho avuto notizia della sua drammatica e rapida malattia solo quando, purtroppo, non c'era più. Dalla moglie Franca ho poi saputo dei suoi ultimi drammatici mesi e credo non ci siano parole per descriverli. Non aver potuto essergli vicino in quel terribile periodo ha reso più forte il mio dolore e il mio rammarico.

Questo brevissimo ricordo per dire che ho perso un grande Amico, ma certamente resterà sempre nel mio cuore il carissimo ricordo di Amedeo e l'affetto per la sua famiglia. 
Chi ha conosciuto Amedeo o, ancora meglio, chi ci ha lavorato o collaborato troverà queste mie semplici parole tanto riduttive quanto scontate.

Il Dottor Amedeo De Vecchi, senza tema di smentita, è stato tra gli antesignani di questa tecnica dialitica, e questa tecnica dialitica deve qualcosa ad Amedeo De Vecchi: non dovremo dimenticarlo. Perciò, alla ripresa di questa indagine (dal 2001 al 2013), è nata spontanea la decisione di dedicarla a Lui.

La sua elevata e indubbia preparazione scientifica ne faceva, secondo me, un "medico speciale". Aveva dalla sua una grande capacità riflessiva, una calma interiore che non so se e quanto di facciata o per un autocontrollo direi magistrale. Aveva anche (e agiva con) un buon senso non comune, una capacità di essere umile e una naturale empatia, che, associati alla facilità di entusiasmarsi e a quanto fin qui scritto, lo rendevano... Amedeo De Vecchi.

Mi ha veramente disturbato e continua a turbarmi la notizia che Amedeo non è più tra noi, nefrologi, pazienti... immagino i suoi familiari.

Sono certo che tutti i Colleghi chiamati a partecipare a questa indagine si sono impegnati anche e proprio per onorare il ricordo di questo Uomo e Collega, che resta tra noi grazie al ricordo che abbiamo e avremo di Lui.

Marco Lombardi 